\title{
Surface carboxylation of cellulose nanowhiskers using MPEG-TEMPO: its recovery and recycling
}

\author{
Jun Araki ${ }^{1,2}$ and Maiko Iida ${ }^{1}$
}

The surface carboxylation of cellulose nanowhiskers (CNWs) prepared by hydrochloric acid hydrolysis was performed via the oxidation of the primary hydroxyl groups on the surface using mPEG2000-TEMPO, a 2,2,6,6-tetramethyl-1-piperidinyloxy radical moiety grafted to monomethoxy poly(ethylene glycol) 2000, to yield carboxylated CNWs (CCNWs). After the oxidation step, the used mPEG2000-TEMPO was recovered by extraction with dichloromethane (DCM) and reused for further oxidation cycles. mPEG2000-TEMPO could be used for the surface oxidation of CNWs for at least three repeated cycles, although the degree of oxidation in the second and third cycles was reduced to $60 \%$ of that in the first cycle. The recovery of mPEG2000-TEMPO after every cycle was not complete ( $80 \%)$, thus suggesting an irreversible adsorption of mPEG2000-TEMPO on the surface of the CCNWs. Soxhlet extraction of the obtained CCNWs with DCM, however, did not achieve a recovery of mPEG2000-TEMPO. Polymer Journal (2016) 48, 1029-1033; doi:10.1038/pj.2016.65; published online 17 August 2016

\section{INTRODUCTION}

Nanosized fibrous (that is, nanofiber-like) crystalline assemblies of cellulose or chitin are undoubtedly important in the field of materials chemistry. ${ }^{1-3}$ Cellulose and chitin are natural linear polysaccharides that are abundant in plant and algae cell walls and in the outer shells of crustaceans and insects, respectively. Cellulose possesses a relatively simple chemical structure of $\beta$ - $(1,4)$-polymerized glucose residues, whose innate structure inevitably yields strong networks of intramolecular and intermolecular hydrogen bonds, thus making the main chain of cellulose rigid and inflexible. ${ }^{4-6}$ This chain rigidity allows independent cellulose chains to associate and bundle into fine crystalline nanofibers, called microfibrils, by intermolecular constraints through systematic hydrogen bonds. ${ }^{5,6}$ Effective treatments to release these constraints by interfibrillar oxidation ${ }^{2}$ or shearing under acidic conditions $s^{3}$ have enabled the isolation of these microfibrils with curious morphologies, such as extremely high aspect ratios, into nanofiber dispersions. These nanofibers possess a number of attractive features, including a high degree of crystallinity, outstanding mechanical properties relative to their low densities, low thermal expansions, non-toxicity and biocompatibility, and low environmental impact. The utilization of these ideal nanofibers to create novel materials with many potential applications has been extensively examined in previous studies. ${ }^{2,3,7-10}$

Although a great number of trials for direct extraction of the high aspect-ratio microfibrils have been examined in this century, fragmentation treatments of these microfibrils by mineral acid hydrolysis have been traditionally employed to yield shortened crystalline rod-like particles known as 'nanowhiskers'., 1,5,6 These nanowhiskers, which have advantages similar to those of nanofibers described above, have been utilized for many applications, including in food additives, cosmetics, tableting of medicines and heat-resistant slabs; ${ }^{11}$ and their fundamental properties such as flow properties ${ }^{12-17}$ and liquid crystal formation ${ }^{18-21}$ have been investigated in detail.

The good dispersion of a cellulose nanowhisker (CNW) suspension may be achieved in several ways: under specific conditions, such as an aqueous electrolyte solution or apolar organic solvents, steric stabilization by surface-adsorbed surfactants or surface-grafted polymers is required. ${ }^{22-26}$ Otherwise, aqueous suspensions of these nanowhiskers remain stable without sedimentation due to the electrostatic repulsions generated by surface charge groups, even with a moderate concentration of electrolytes. ${ }^{1}$ A considerable amount of sulfate groups introduced on the surface of CNW particles during sulfuric acid hydrolysis is responsible for their dispersion stability. ${ }^{16,17,19,27,28}$ Conversely, when no sulfate groups are introduced on CNWs via hydrochloric acid hydrolysis, the CNW dispersion has limited stability and easily aggregates with the addition of a drop of electrolyte solution. ${ }^{15,16,22}$ Because many properties of CNW suspensions, such as viscosity ${ }^{16,17}$ and liquid crystal formation, ${ }^{20,21}$ are drastically affected by the amount of surface charge groups, a technique that enables the controlled introduction of these charge groups is important for managing the stability of the system and/or the abovementioned suspension properties.

Several methods have been reported to be effective for controlling the amount of surface charge on CNWs, including variations in the hydrolysis temperatures and the reaction duration ${ }^{28}$ or desulfation of sulfated CNWs using alkaline hydrolysis of surface sulfate groups. ${ }^{29,30}$ Another series of methods involves the post-modification of surface hydroxyl groups of sulfate-free CNWs prepared by hydrochloric acid

\footnotetext{
${ }^{1}$ Department of Chemistry and Materials, Faculty of Textile Science and Technology, Shinshu University, Nagano, Japan and ${ }^{2}$ Division of Biological and Medical Fibers, Institute for Fiber Engineering (IFES), Interdisciplinary Cluster for Cutting Edge Research (ICCER), Shinshu University, Nagano, Japan Correspondence: Dr J Araki, Faculty of Textile Science and Technology, Shinshu University, Tokida 3-15-1, Ueda, 386-8567 Nagano, Japan. E-mail: jun@shinshu-u.ac.jp

Received 1 April 2016; revised 1 June 2016; accepted 2 June 2016; published online 17 August 2016
} 
hydrolysis; by using the CNWs as a starting material, the controlled introduction of sulfate groups, ${ }^{17}$ phosphate groups, ${ }^{31}$ 2-hydroxy-3trimethylammoniopropyl (that is, quaternary ammonium) groups ${ }^{30,32}$ or carboxyl groups ${ }^{22}$ gave well-dispersed CNW suspensions. Besides the above methods, surface carboxylation mediated by 2,2,6, 6-tetramethyl-1-pipridinyloxy radical (TEMPO) 22 is superior to others in several aspects, including considerably mild aqueous reaction conditions $\left(0^{\circ} \mathrm{C}-\right.$ r.t., $\left.\mathrm{pH}=10-11\right)$, providing the widest control of introduced carboxyl amounts, and no possibility of removing the introduced carboxyls, which is different from the hydrolytic cleavage observed for sulfates or phosphates.

An additional advantage of TEMPO-mediated carboxylation is the trace catalytic amount of TEMPO that is required for oxidation because of its participation in oxidation as a cycle of nitroxyl radicals, hydroxylamines and nitrosonium ion forms. ${ }^{2,33}$ Consequently, TEMPO can be reused after recovery from the oxidation systems. Such attempts have actually been realized by the use of various types of recoverable TEMPO, including TEMPO supported on solid particles such as silica gel ${ }^{34}$ or magnetite nanoparticles, ${ }^{35-37}$ sol-gel entrapped TEMPO, ${ }^{38}$ polymerized TEMPO $^{39}$ and TEMPO grafted on the terminals of linear polymers, ${ }^{40-43}$ all of which were reported to be readily recoverable and reusable after the oxidation of various low-molecular weight substances. Such reuse of TEMPO is attractive from an economical and environmental viewpoint because it might avoid the leakage of oxidants to wastewater.

In this study, we examined the use of mPEG2000-TEMPO, which is a TEMPO reagent grafted to a terminal of monomethoxy poly (ethylene glycol) with molecular weight of 2000 (mPEG2000), for the surface oxidation of CNWs to yield carboxylated CNWs (CCNWs). The water-soluble mPEG2000-TEMPO may be ideal for the production of CCNWs under mild aqueous conditions and subsequent recovery. Furthermore, an easy separation of the mPEG2000-TEMPO from CCNWs is expected by centrifugation or filtration because the latter are solid. The investigation includes the recovery of mPEG2000-TEMPO used for the carboxylation of CNWs by centrifugation and subsequent extraction with dichloromethane (DCM), changes in the degree of oxidation and mPEG2000-TEMPO recovery and characterization of the obtained CCNWs by means of Fourier-transform infrared (FTIR) spectroscopy, electron microscopy and conductometric titration for determining the carboxyl content on the surface. To the best of our knowledge, this study is the first trial of the use of recoverable TEMPO for the surface oxidation (that is, solid surface modification) of CNWs.

\section{MATERIALS AND METHODS}

\section{Materials}

Whatman CF11 cellulose powder (Whatman International Ltd., Maidstone, Kent, UK) was used as a cellulose source. mPEG2000 with a number average molecular weight of 2000 was purchased from Sigma-Aldrich Co. (St Louis, MO, USA). All other reagents were purchased from Wako Pure Chemical Industries, Ltd. (Osaka, Japan). DCM and dimethylformamide were distilled from calcium hydride and stored over $4 \mathrm{~A}$ molecular sieves. Trioctylamine was dried over $\mathrm{KOH}$ pellets. All other reagents were used without further purification. The concentration of purchased aqueous sodium hypochloride $(\mathrm{NaClO})$ solution was determined as $10.5 \mathrm{wt} \%$ (note that this value is not the available chlorine content but the actual weight ratio of $\mathrm{NaClO}$ ) by iodometry. 22

\section{Preparation of CNWs by hydrochloric acid hydrolysis}

CNWs were prepared following the method reported in a previous study. ${ }^{22}$ In brief, $10 \mathrm{~g}$ of CF11 was added to a boiling solution of $\mathrm{HCl}(2.5 \mathrm{M}, 100 \mathrm{ml})$ and refluxed for $15 \mathrm{~min}$. The sample was then diluted with $100 \mathrm{ml}$ of water, filtered and washed thoroughly with water by suction until neutral. The washed hydrolysate was dispersed in water at ca. $10 \%$ concentration and subjected to homogenization using a Waring-type blender for $40 \mathrm{~min}(10 \mathrm{~min} \times 4$ times). Coarse unground particles were separated by gentle centrifugation a few times (3100 r.p.m., $5 \mathrm{~min}$ ), and the combined turbid supernatants containing CNWs were dialyzed against deionized water overnight to yield a purified $\mathrm{CNW}$ suspension of $0.5-1 \%$ solid content. The solid yield was $\sim 40 \%$. A higher solid content in the suspensions could be obtained by adding a trace amount of $\mathrm{HCl}$ (a few drops of $1 \mathrm{M} \mathrm{HCl}$ ) to the dilute suspension to facilitate the sedimentation of CNWs, gentle centrifugation, discarding of transparent supernatant and dialysis.

\section{Synthesis of mPEG2000-TEMPO}

As described in previous studies, mPEG2000-TEMPO was synthesized by the mesylation of the terminal hydroxyl groups of mPEG2000 $0^{44,45}$ and subsequent etherification with the sodium alcoholate of 4-hydroxy-2,2,6,6-tetramethyl-1piperidinyloxy, radical (4-OH-TEMPO). ${ }^{40}$ mPEG2000 (5 g, $2.5 \mathrm{mmol}$, dried in vacuo at $40^{\circ} \mathrm{C}$ overnight) and trioctylamine $(5.49 \mathrm{ml}, 12.5 \mathrm{mmol})$ were dissolved in $100 \mathrm{ml}$ of anhydrous dimethylformamide. The solution was cooled in an ice bath, and methanesulfonyl chloride $(0.97 \mathrm{ml}, 12.5 \mathrm{mmol})$ was added dropwise with stirring, followed by further stirring at room temperature for $24 \mathrm{~h}$. After the solvent was evaporated to dryness, the residue was dissolved in a minimum amount of water, and the aqueous solution was extracted with DCM $(50 \mathrm{ml} \times 3)$. The combined organic layers were again concentrated using a rotary evaporator and poured into cold diethyl ether to precipitate the product, mPEG2000-OMs, which was collected by filtration and dried in vacuo to obtain a white solid with $91 \%$ yield.

4-OH TEMPO (155 mg, $0.9 \mathrm{mmol}$ ) was added to a dispersion of sodium hydride (assay $60 \%, 36 \mathrm{mg}$ of oil dispersion, $0.9 \mathrm{mmol}$ ) in anhydrous dimethylformamide $(15 \mathrm{ml})$ in a Schlenk tube and stirred at room temperature for $1 \mathrm{~h}$ under an argon atmosphere. mPEG2000-OMs (450 mg, $0.225 \mathrm{mmol}$ ) were added to the solution and stirred at room temperature for $24 \mathrm{~h}$. A transparent orange solution obtained after the filtration of the reaction mixture was evaporated to dryness to give an orange residue. This residue was redissolved in DCM $(2 \mathrm{ml})$ and poured into diethyl ether $(100 \mathrm{ml})$ to precipitate the product, mPEG2000-TEMPO, which was collected by filtration and vacuum-dried to give an orange solid in $90 \%$ yield. The degree of modification of mPEG2000 terminal hydroxyl groups was found to be $62 \%$ by ${ }^{1} \mathrm{H} \mathrm{NMR} \mathrm{in} \mathrm{CDCl}_{3}$.

\section{Surface oxidation of CNWs using mPEG2000-TEMPO}

A typical procedure for CNW oxidation using mPEG2000-TEMPO is as follows: in a CNW suspension (50 g, $1.23 \mathrm{wt} \%$, containing $615 \mathrm{mg} \mathrm{CNWs}$ ), $\mathrm{NaBr}$ (500 mg) and mPEG2000-TEMPO (500 mg, containing $1.46 \times 10^{-4} \mathrm{~mol}$ of the TEMPO moiety) were dissolved sequentially. The oxidation was initiated by the addition of an aqueous $\mathrm{NaClO}$ solution $(5.86 \mathrm{ml}$, containing $615 \mathrm{mg}$ $\mathrm{NaClO}$ ) and was continued at room temperature for $4 \mathrm{~h}$, keeping the system $\mathrm{pH}$ at $10-11$ with the dropwise addition of $2 \mathrm{M} \mathrm{NaOH}$. After quenching the oxidation with the addition of $0.5 \mathrm{ml}$ of ethanol, $2 \mathrm{ml}$ of aqueous $1 \mathrm{M} \mathrm{CaCl} 2$ solution was added to facilitate aggregation of the obtained CCNWs, followed by gentle centrifugation (3100 r.p.m., 5-10 $\mathrm{min}$ ) to separate the CCNWs from the supernatant containing mPEG2000-TEMPO. The collected CCNWs pellet was redispersed in $0.1 \mathrm{M} \mathrm{HCl}$, centrifuged and washed, and the cycle was repeated three times to convert the surface carboxyl groups to their acid form. Final dialysis of the collected pellet against deionized water resulted in a stable suspension of CCNWs. The supernatant fractions containing $\mathrm{CaCl}_{2}$ and $\mathrm{HCl}$ were combined and subjected to the procedure described in the Section 'Recovery of mPEG2000-TEMPO from the reaction system' for recovery of mPEG2000-TEMPO, which was used for further CNWs oxidation. In every reaction cycle, the weight ratio of mPEG2000-TEMPO to CNWs was kept constant. In other words, when the recycling of mPEG2000-TEMPO was considered, the first and second reaction cycles were scaled up appropriately to reserve a sufficient amount of mPEG2000-TEMPO for the subsequent cycles, considering the decreases in mPEG2000-TEMPO recovery. Detailed oxidation conditions using the recovered mPEG2000-TEMPO are described in the Supplementary Information. 
Recovery of mPEG2000-TEMPO from the reaction system

The used mPEG2000-TEMPO was extracted from the aqueous supernatant with DCM (typically $100 \mathrm{ml} \times 3$ for $200 \mathrm{ml}$ of supernatant). The combined organic layers were dried with anhydrous sodium sulfate, filtered and concentrated with a rotary evaporator to recover mPEG2000-TEMPO as a dark orange solid.

\section{Characterizations}

The ${ }^{1} \mathrm{H}$ NMR spectra at $400 \mathrm{MHz}$ were recorded in $\mathrm{CDCl}_{3}$ using a Bruker AVANCE spectrometer (Bruker BioSpin KK, Yokohama, Japan) at room temperature. The chemical shifts were referenced to tetramethylsilane. Surface carboxyl group content of the CCNWs was determined by conductometric titration, according to a previous study, ${ }^{16}$ with a slight modification. A mixture of an aqueous CCNW suspension (typically containing ca. $300 \mathrm{mg}$ of CCNWs) and $0.1 \mathrm{M}$ aqueous $\mathrm{HCl}(1 \mathrm{ml})$ was titrated using $0.1 \mathrm{M}$ aqueous $\mathrm{NaOH}$ at an addition rate of $0.02 \mathrm{ml} / 30 \mathrm{~s}$. The conductivity and $\mathrm{pH}$ values of the system
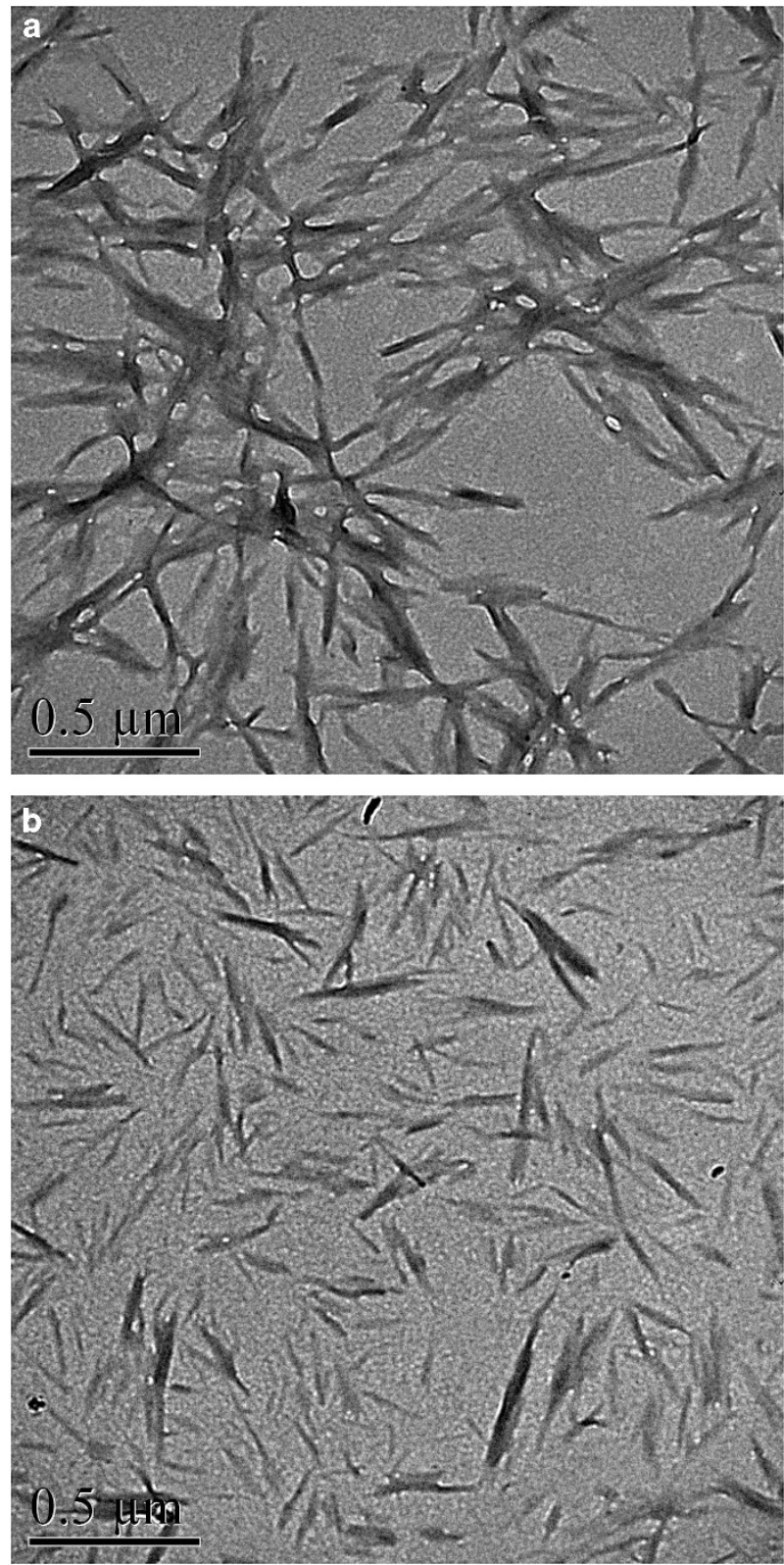

Figure 1 Electron micrographs of (a) CNWs and (b) CCNWs obtained in the third carboxylation cycle using recovered mPEG2000-TEMPO. were simultaneously monitored using a $\mathrm{pH} /$ conductivity meter (Seven Go Duo-SG78, METTLER TOLEDO, OH, USA). Attenuated total reflectance FTIR (ATR-FTIR) measurements of the freeze-dried samples were obtained using a Shimadzu IRPrestage-21 spectrometer (Shimadzu Corporation, Kyoto, Japan), equipped with a diamond ATR accessory (SensIR Technologies DurasampleIR II, Smiths Detection, UK), in air at $4 \mathrm{~cm}^{-1}$ resolution and 32 scans. The sizes and shapes of the CNWs and CCNWs were observed using transmission electron microscopy (JEOL JEX-2100, JEOL Ltd., Tokyo, Japan). A drop of very dilute aqueous suspensions of the samples was deposited on a grid covered with a carbon-coated Formvar film that had been treated with $0.2 \%$ aqueous bacitracin solution. After drying, the samples were observed at $80 \mathrm{kV}$ using the defocus-contrast technique.

\section{RESULTS AND DISCUSSION}

Oxidation of CNWs using mPEG2000-TEMPO and characterization of the obtained CCNWs

mPEG2000-TEMPO used in the present study was synthesized via the mesylation of terminal hydroxyl groups in mPEG2000 and subsequent etherification with sodium alcoholate of 4-OH-TEMPO, as reported in a previous study (Ferreira et al. ${ }^{40}$ ). Because the obtained mPEG2000TEMPO is a versatile TEMPO derivative that is soluble in various types of solvents, including water, it can be utilized for surface oxidation of CNWs, which is conducted in an aqueous solution. ${ }^{22}$ As described in Materials and methods, the authors examined the surface carboxylation of CNWs using mPEG2000-TEMPO under conditions similar to those of the conventional oxidation using free TEMPO: a comparable TEMPO moiety concentration (ca. $5 \mathrm{wt} \%$ ) and an identical amount of $\mathrm{NaClO}$ (equal weight with $\mathrm{CNWs}$ ). The reaction system indicated a gradual decay in $\mathrm{pH}$, which is an indication of successful carboxylation. After the reaction, aggregations generated by the addition of electrolyte $\left(\mathrm{CaCl}_{2}\right.$ and $\left.\mathrm{HCl}\right)$ were collected by repeated gentle centrifugation cycles, followed by dialysis, which yielded a turbid and stable suspension of CCNWs.

Electron micrographs of CNWs and CCNWs are shown in Figure 1. The CCNW particles shown in Figure $1 \mathrm{~b}$ have a morphology of $5-10 \mathrm{~nm}$ in width and $100-150 \mathrm{~nm}$ in length, which are comparable to those obtained by previously described TEMPO-oxidized CCNWs. ${ }^{22}$ Figure 1 also clearly indicates a far better dispersion of the charged CCNWs relative to charge-free CNWs, which have been already reported to form loose aggregations due to their lack of surface charge. ${ }^{16}$ Figure 2 shows the ATR-FTIR spectra of CNWs and CCNWs. The spectrum of the CCNWs clearly exhibits absorption at $1726 \mathrm{~cm}^{-1}$, which is absent in the spectrum of the CNWs and is attributed to the presence of surface carboxyl groups. Almost no other significant changes were observed in the two spectra.

The abovementioned results, which are almost identical to those observed for the CCNWs obtained by free TEMPO-mediated oxidations in the previous study, ${ }^{22}$ clearly indicate a successful oxidation of the primary hydroxyl groups on the surface of the CNWs into carboxyl groups using mPEG2000-TEMPO.

\section{Carboxyl content of the CCNWs by repeated surface oxidation using mPEG2000-TEMPO}

The mPEG2000-TEMPO used in the present study was originally developed to be used repeatedly after its recovery from the system. The typical procedure for its recovery is direct precipitation from the reaction solution with diethyl ether and subsequent filtration, thus enabling a separation from the ethereal solution containing oxidized low-molecular weight products ${ }^{41-43}$ or extraction with DCM. ${ }^{40}$ In the present investigation of oxidation of CNWs in an aqueous system, the latter method seems to work efficiently; namely, the facile recovery of mPEG2000-TEMPO is expected because of separation of the 
supernatant containing mPEG2000-TEMPO and other inorganic salts from the solid CCNWs by means of centrifugation or filtration and its subsequent extraction with DCM. We examined the recovery of mPEG2000-TEMPO by applying this strategy, the details of which are described in Materials and methods and Scheme 1. The recovered mPEG2000-TEMPO was used in subsequent runs to investigate the changes in recovery and degree of carboxylation.

Table 1 summarizes the yields of CCNWs, recovery of mPEG2000TEMPO and surface carboxyl contents of the obtained CCNWs based on the oxidation cycle of the mPEG2000-TEMPO. The carboxyl content of the CCNWs obtained in the first cycle, $5.54 \times 10^{-4}$ $\mathrm{mol} \mathrm{g}^{-1}$, was relatively lower than the value for the CCNWs obtained with free-TEMPO oxidation, $9.15 \times 10^{-4} \mathrm{molg}^{-1}$, ${ }^{22}$ but was still comparable. However, in the second and third oxidation cycles, a certain degree of decrease in carboxyl content was observed; it was ca. $60 \%$ of the value in the first cycle. There could be several reasons for this low degree of oxidation. A previous study using mPEG-TEMPO with an identical chemical structure to ours indicated a similar decrease in oxidation efficiency by the recovered mPEG-TEMPO. Although an 'occlusion' of the terminal TEMPO moiety in mPEG2000-TEMPO during the precipitation step with diethyl ether
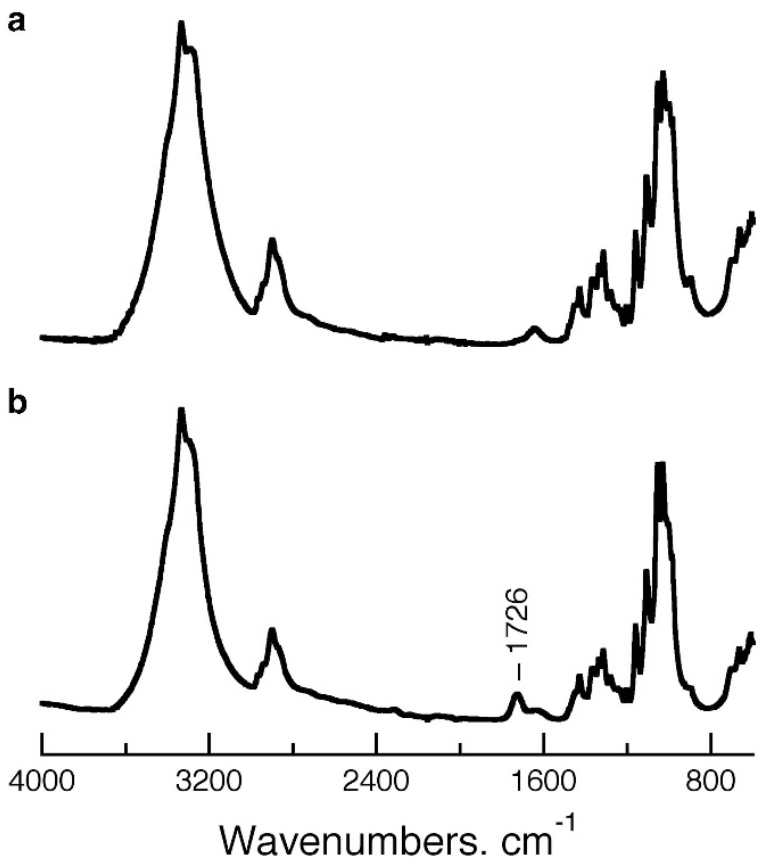

Figure 2 ATR-FTIR spectra of (a) CNWs and (b) CCNWs obtained by carboxylation using new mPEG2000-TEMPO.

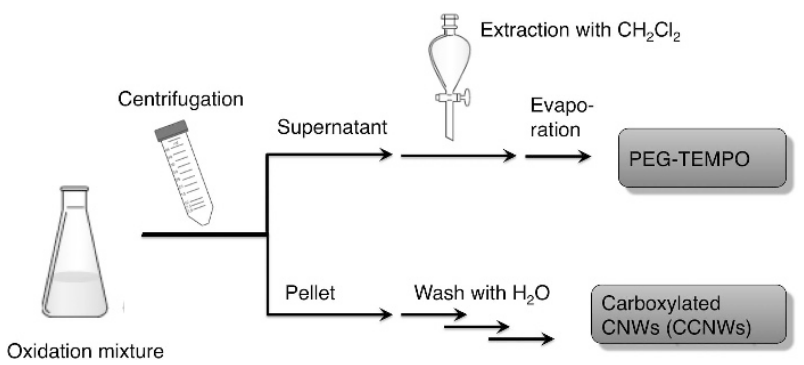

Scheme 1 Experimental scheme of mPEG2000-TEMPO recovery from the reaction mixture of $\mathrm{CNW}$ oxidation. A full color version of this scheme is available at Polymer Journal online. was speculated to be a reason for the observed low oxidation efficiency, this reason cannot explain our observations because we did not employ ether-based precipitation for the recovery of mPEG2000-TEMPO. In addition, such an occlusion should have no effect on the second cycle or thereafter because the recovered mPEG2000-TEMPO is completely dissolved again in the reaction mixture. Unfortunately, it is not clear why there was a decrease in the oxidation efficiency when using recovered mPEG2000-TEMPO, although the reagent could be used to introduce carboxyl groups that are comparable to those obtained using free TEMPO.

Table 1 also summarizes the recovered amounts of mPEG2000TEMPO after each oxidation cycle. All cycles present similar levels of recovery, $\sim 80 \%$, which is slightly lower than those found in previous investigations on recoverable polymeric TEMPO, ${ }^{34,35,40,42}$ which showed an almost quantitative recovery of the polymeric TEMPOs. Although our recovery is also satisfactory, our experiments should be further refined to improve the recovery of MPEG2000-TEMPO after CCNW oxidation. The irreversible adsorption of mPEG2000-TEMPO onto the CCNWs may be one of the reasons for the observed low recovery of the mPEG2000-TEMPO. We sought to confirm this hypothesis by Soxhlet extraction of the obtained CCNWs with DCM for $48 \mathrm{~h}$. A miniscule amount of solid $(44 \mathrm{mg}$ ) was extracted from $372 \mathrm{mg}$ of the CCNWs obtained in the first cycle, although no trace of mPEG was observed in this solid by ${ }^{1} \mathrm{H}$ NMR (data not shown). Furthermore, as shown in Figure 2, the FTIR spectrum of the CCNWs was almost identical to that of the CNWs, except for an absorption that was attributed to carbonyl stretching; namely, the former indicated no absorption characteristic of crystalline and/or amorphous PEG. These results also show that the obtained CCNWs are not contaminated with PEG or any other impurities. Although the above two results imply that the adsorption of mPEG2000-TEMPO on CCNWs is implausible, very recent publications have shown the strong adsorption of PEG onto CNWs by only mixing and subsequent freeze drying, which resulted in the formation of polymer brushes working effectively for steric repulsions. ${ }^{46,47}$ We still speculate that one of the reasons for the low mPEG2000-TEMPO recovery is its adsorption on CCNWs. Other reasons are still unclear at present and should be elucidated in the future. The recovery of mPEG2000TEMPO in the present examination is required as minimum for repeated uses.

\section{CONCLUSION}

The surface carboxylation of CNWs to yield CCNWs was successfully achieved using mPEG2000-TEMPO. The mPEG2000-TEMPO was recovered and reused at least three times by extracting it from the reaction supernatant using DCM. The degree of oxidation in the

Table 1 Yields of CCNWs, mPEG2000-TEMPO recovery and surface carboxyl contents of the obtained CCNWs with the oxidation cycle of mPEG2000-TEMPO

\begin{tabular}{|c|c|c|c|}
\hline $\begin{array}{l}\text { Oxidation cycle of } \\
\text { MPEG2000-TEMPO }\end{array}$ & $\begin{array}{c}\text { CCNW } \\
\text { yields, \% }\end{array}$ & $\begin{array}{c}\text { Recovery of } \\
\text { mPEG2000-TEMPO, } \\
\%^{\mathrm{a}}\end{array}$ & $\begin{array}{c}\text { Surface carboxyl content, } \\
\quad \times 10^{-4} \mathrm{~mol} \mathrm{~g}^{-1 \mathrm{~b}}\end{array}$ \\
\hline 1 & 91.7 & 80.0 & $5.54 \pm 0.25$ \\
\hline 2 & 94.7 & 82.8 & $3.40 \pm 0.39$ \\
\hline 3 & 96.1 & 76.5 & $3.48 \pm 0.25$ \\
\hline
\end{tabular}

Abbreviations: CCNWs, carboxylated CNWs; CNWs, cellulose nanowhiskers; mPEG2000, monomethoxy poly(ethylene glycol) 2000; TEMPO, 2,2,6,6-tetramethyl-1-pipridinyloxy radical. aRatio against feeding in each cycle.

${ }^{b}$ Carboxyl contents of starting (unoxidized) CNWs were $1.70 \times 10^{-5} \mathrm{~mol} \mathrm{~g}^{-1}$ 
second and third runs, however, was lower than that in the first cycle. The recovery of mPEG2000-TEMPO was low, at ca. $80 \%$ after every oxidation cycle. Although an irreversible adsorption of mPEG2000TEMPO may be responsible for the low recovery, the main reason for this trend remains unclear.

The current work presents a favorable method for carboxylation comparable to the free-TEMPO oxidation of CNWs. This method shows more facile mPEG2000-TEMPO recovery from the oxidation system compared with that of free TEMPO. The remaining problems, including the relatively lower degree of oxidation and recovery, render the present method unsuitable as a first-line preparation method for CCNWs. To overcome these difficulties, other types of recoverable TEMPO, including solid-supported TEMPO ${ }^{34-37}$ and polymerized TEMPO, ${ }^{39}$ should be considered. The synthesis of novel TEMPO derivatives grafted to other water-soluble polymers may also be promising.

\section{CONFLICT OF INTEREST}

The authors declare no conflict of interest.

\section{ACKNOWLEDGEMENTS}

We are grateful to Ms Sachiko Yoshioka (Shinshu University) for FTIR and ${ }^{1} \mathrm{H}$ NMR measurements and to the Satellite Venture Business Laboratories of Shinshu University for the use of an electron microscope.

1 Araki, J. Electrostatic or steric? - Preparations and characterizations of well-dispersed systems containing rod-like nanowhiskers of crystalline polysaccharides. Soft Matter $\mathbf{9}$, 4125-4141 (2013).

2 Isogai, A., Saito, T. \& Fukuzumi, H. TEMPO-oxidized cellulose nanofibers. Nanoscale 3, 71-85 (2011)

3 Ifuku, S. \& Saimoto, H. Chitin nanofibers: Preparations, modifications, and applications. Nanoscale 4, 3308-3318 (2012).

4 Klemm, D., Kramer, F., Moritz, S., Lindström, T., Ankerfors, M., Gray, D. \& Dorris, A Nanocelluloses: a new family of nature-based materials. Angew. Chem. Int. Ed. 50, 5438-5466 (2011).

5 Azizi Samir, M. A. S., Alloin, F. \& Dufresne, A. Review of recent research into cellulosic whiskers, their properties and their application in nanocomposite field. Biomacromolecules 6, 612-626 (2005).

6 de Souza Lima, M. M. \& Borsali, R. Rodlike cellulose microcrystals: structure, properties, and applications. Macromol. Rapid Commun. 25, 771-787 (2004).

7 Habibi, Y., Lucia, L. A. \& Rojas, O. J. Cellulose nanocrystals: chemistry, self-assembly, and applications. Chem. Rev. 110, 3479-3500 (2010).

8 Eichhorn, S. J. Cellulose nanowhiskers: promising materials for advanced applications. Soft Matter 7, 303-315 (2011)

9 Dufresne, A. Polysaccharide nanocrystal reinforced nanocomposites. Can. J. Chem. 86 484-494 (2008).

10 Ramires, E. C. \& Dufresne, A. A review of cellulose nanocrystals and nanocomposites. Tappi J. 10, 9-16 (2011).

11 Battista, O. A. \& Smith, P. A. Microcrystalline cellulose. The oldest polymer finds new industrial uses. Ind. Eng. Chem. 54, 20-29 (1962).

12 Marchessault, R. H., Morehead, F. F. \& Koch, M. J. Some hydrodynamic properties of neutral suspensions of cellulose crystallites as related to size and shape. J. Colloid Sci. 16, 327-344 (1961).

13 Marchessault, R. H., Morehead, F. F. \& Yang, J. T. Some hydrodynamic properties of ramie crystallites in phosphate buffer. J. Colloid Sci. 16, 345-360 (1961).

14 Hermans, J. Flow of gels of cellulose microcrystals. I. Random and liquid crystalline gels. J. Polym. Sci. C 2, 129-144 (1963).

15 Hermans, J. Flow of gels of cellulose microcrystals. II. Effect of added electrolyte. J. Polym. Sci. C 2, 145-152 (1963).

16 Araki, J., Wada, M., Kuga, S. \& Okano, T. Flow properties of microcrystalline cellulose suspension prepared by acid treatment of native cellulose. Colloids Surf. A 142, 75-82 (1998).

17 Araki, J., Wada, M., Kuga, S. \& Okano, T. Influence of surface charge on viscosity behavior of cellulose microcrystal suspension. J. Wood Sci. 45, 258-261 (1999).

18 Marchessault, R. H., Morehead, F. F. \& Koch, M. J. Liquid crystal systems from fibrillar polysaccharides. Nature 184, 632-633 (1959).
19 Revol, J.-F., Bradford, H., Giasson, J., Marchessault, R. H. \& Gray, D. G. Helicoidal selfordering of cellulose microfibrils in aqueous suspension. Int. J. Biol. Macromol. 14, 170-172 (1992).

20 Araki, J., Wada, M., Kuga, S. \& Okano, T. Birefringent glassy phase of a cellulose microcrystal suspension. Langmuir 16, 2413-2415 (2000).

21 Araki, J. \& Kuga, S. Effect of trace electrolyte on liquid crystal type of cellulose microcrystals. Langmuir 17, 4493-4496 (2001).

22 Araki, J., Wada, M. \& Kuga, S. Steric stabilization of a cellulose microcrystal suspension by poly(ethylene glycol) grafting. Langmuir 17, 21-27 (2001).

23 Araki, J., Kuga, S. \& Magoshi, J. Influence of reagent addition on carbodiimide mediated amidation for poly(ethylene glycol) grafting. J. Appl. Polym. Sci. 85, 1349-1352 (2002).

24 Araki, J. \& Kurihara, M. Preparation of sterically stabilized chitin nanowhisker dispersions by grafting of poly(ethylene glycol) and evaluation of their dispersion stability. Biomacromolecules 16, 379-388 (2015).

25 Araki, J. \& Mishima, S. Steric stabilization of 'charge-free' cellulose nanowhiskers by grafting of poly(ethylene glycol). Molecules 20, 169-184 (2015).

26 Heux, L., Chauve, G. \& Bonini, C. Nonflocculating and chiral-nematic self-ordering of cellulose microcrystals suspensions in nonpolar solvents. Macromolecules 16 8210-8212 (1999).

27 Dong, X. M., Kimura, T., Revol, J.-F. \& Gray, D. G. Effects of ionic strength on the isotropic-chiral nematic phase transition of suspensions of cellulose crystallites. Langmuir 12, 2076-2082 (1996).

28 Dong, X. M., Revol, J.-F. \& Gray, D. G. Effect of microcrystallite preparation conditions on the formation of colloid crystals of cellulose. Cellulose 5, 19-32 (1997).

29 Kloser, E. \& Gray, D. G. Surface grafting of cellulose nanocrystals with poly(ethylene oxide) in aqueous media. Langmuir 26, 13450-13456 (2010).

30 Hasani, M., Cranston, E. D., Westman, G. \& Gray, D. G. Cationic surface functionalization of cellulose nanocrystals. Soft Matter 4, 2238-2244 (2008).

31 Araki, J., Wada, M., Kuga, S. \& Okano, T. in Hydrocolloids. Part 1. Physical Chemistry and Industrial Application of Gels, Polysaccharides, and Proteins (ed. Nishinari, K.) 283-288 (Elsevier Science, The Netherlands, 2000).

32 Zaman, M., Xiao, H., Chibante, F. \& Ni, Y. Synthesis and characterization of cationically modified nanocrystalline cellulose. Carbohydr. Polym. 89, 163-170 (2012).

33 Bragd, P. L., van Bekkum, H. \& Besemer, A. C. TEMPO-mediated oxidation of polysaccharides: survey of methods and applications. Top Catal 27, 49-66 (2004).

34 Fey, T., Fischer, H., Bachmann, S., Albert, K. \& Bolm, C. Silica-supported TEMPO catalysts: synthesis and application in the Anelli oxidation of alcohols. J. Org. Chem. 66, 8154-8159 (2001).

35 Karimi, B. \& Farhangi, E. A highly recyclable magnetic core-shell nanoparticlesupported tempo catalyst for efficient metal- and halogen-free aerobic oxidation of alcohols in water. Chem. Eur. J. 17, 6056-6060 (2011).

36 Schätz, A. Grass, R. N. Stark, W. J. \& Reiser, O. TEMPO supported on magnetic c/co-nanoparticles: a highly active and recyclable organocatalyst. Chem. Eur. J. 14, 8262-8266 (2008).

37 Tucker-Schwartz, A. K. \& Garrell, R. L. Simple preparation and application of TEMPO-coated Fe304 superparamagnetic nanoparticles for selective oxidation of alcohols. Chem. Eur. J. 16, 12718-12726 (2010).

38 Ciriminna, R., Blum, J., Avnir, D. \& Pagliaro, M. Sol-gel entrapped TEMPO for the selective oxidation of methyl $\alpha$-D-glucopyranoside. Chem. Commun. 1441-1442 (2000).

39 Dijksman, A., Arends, I. W. C. E. \& Sheldon, R. A. Polymer immobilised TEMPO (PIPO): an efficient catalyst for the chlorinated hydrocarbon solvent-free and bromide-free oxidation of alcohols with hypochlorite. Chem. Commun. 271-272 (2000).

40 Ferreira, P., Phillips, E., Rippon, D., Tsang, S. C. \& Hayes, W. Poly(ethylene glycol)supported nitroxyls: branched catalysts for the selective oxidation of alcohols. J. Org. Chem. 69, 6851-6859 (2004).

41 Ferreira, P., Phillips, E., Rippon, D. \& Tsang, S. C. Catalytic oxidation of alcohols using molecular oxygen mediated by poly(ethylene glycol)-supported nitroxyl radicals. Appl. Catal. B Environ. 61, 206-211 (2005).

42 Pozzi, G., Cavazzini, M., Quici, S., Benaglia, M. \& Dell'Anna, G. Poly(ethylene glycol)supported TEMPO: an efficient, recoverable metal-free catalyst for the selective oxidation of alcohols. Org. Lett. 6, 441-443 (2004).

43 Benaglia, M., Puglisi, A., Holczknecht, O., Quici, S. \& Pozzi, G. Aerobic oxidation of alcohols to carbonyl compounds mediated by poly(ethylene glycol)-supported TEMPO radicals. Tetrahedron $61,12058-12064$ (2005).

44 Benaglia, M., Cinquini, M. \& Cozzi, F. Improved procedure for the purification ofpeg bound molecules by the use of trioctylamine. Tetrahedron 40, 2019-2020 (1999).

45 Fishman, A., Farrah, M. E., Zhong, J.-H., Paramanantham, S., Carrera, C. \& Lee-Ruff, E. Synthesis and investigation of novel branched peg-based soluble polymer supports. J. Org. Chem. 68, 9843-9846 (2003).

46 Cheng, D., Wen, Y., Wang, L., An, X., Zhu, X. \& Ni, Y. Adsorption of polyethylene glyco (PEG) onto cellulose nano-crystals to improve its dispersity. Carbohydr. Polym. 123, 157-163 (2015).

47 Bardet, R., Belgacem, N. \& Bras, J. Flexibility and color monitoring of cellulose nanocrystal iridescent solid films using anionic or neutral polymers. ACS Appl. Mater. Interfaces 7, 4010-4018 (2015).

Supplementary Information accompanies the paper on Polymer Journal website (http://www.nature.com/pj) 